PROFESIONALES Y HERRAMIENTAS PARA EL DESARROLLO LOCAL Y SUS SINERGIAS TERRITORIALES. EVALUACIÓN Y PROPUESTAS DE FUTURO IX Coloquio Nacional de Desarrollo Local del GTDL-AGE 

ANTONIO MARTÍNEZ PUCHE, XAVIER AMAT MONTESINOS, ISABEL SANCHO CARBONELL y DANIEL SANCHIZ CASTAÑO (EDS.)

\section{PROFESIONALES Y HERRAMIENTAS PARA EL DESARROLLO LOCAL Y SUS SINERGIAS TERRITORIALES. EVALUACIÓN Y PROPUESTAS DE FUTURO}

IX Coloquio Nacional de Desarrollo Local del GTDL-AGE

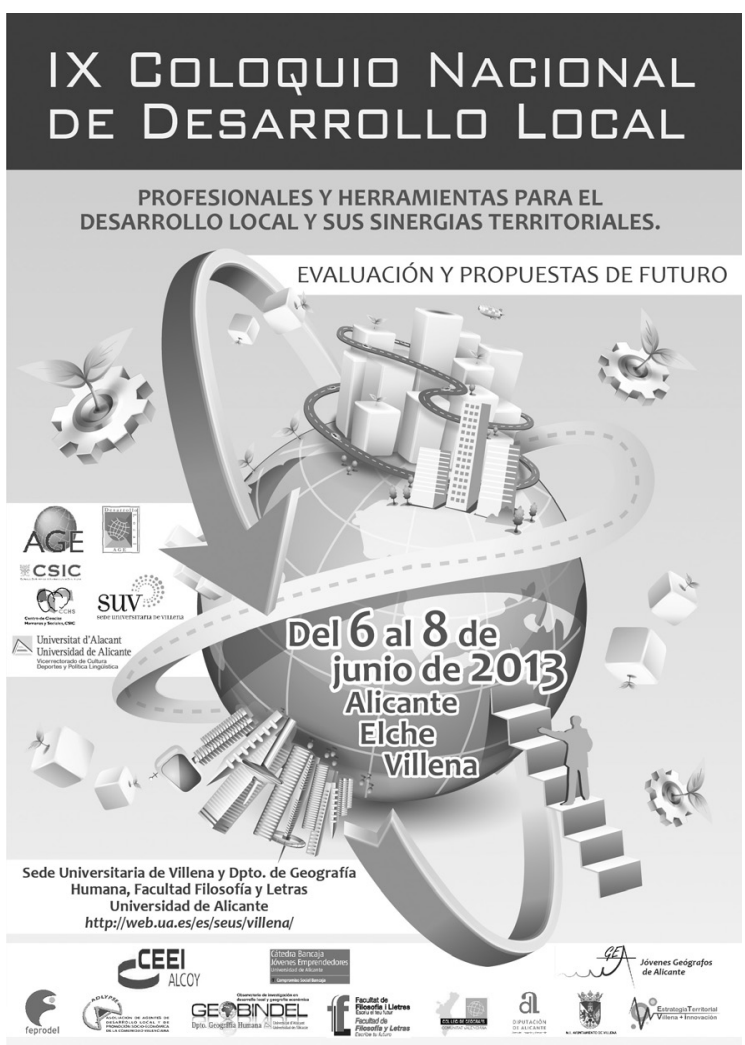


Este libro ha sido examinado y valorado por evaluadores ajenos a la Universidad de Alicante, con el fin de garantizar la calidad científica del mismo.

Publicacions de la Universitat d'Alacant

03690 Sant Vicent del Raspeig

Publicaciones@ua.es

http://publicaciones.ua.es

Telèfon: 965903480

(C) Antonio Martínez Puche, Xavier Amat Montesinos,

Isabel Sancho Carbonell y Daniel Sanchiz Castaño (eds.), 2016

(C) d'aquesta edició: Universitat d'Alacant

ISBN: 978-84-16724-00-0

Dipòsit legal: A 92-2016

Disseny de coberta: candela ink

Composició: Página Maestra (Miguel Ángel Sánchez Hernández)

Impressió i enquadernació: Guada Impresores

\section{unte \\ Unión de Editoriales
Universitarias Españolas \\ WWW.une.es
WWA}

Esta editorial es miembro de la UNE, cosa que garantiza la difusión y comercialización nacional y internacional de sus publicaciones.

Reservados todos los derechos. Cualquier forma de reproducción, distribución, comunicación pública o transformación de esta obra sólo puede ser realizada con la autorización de sus titulares, salvo excepción prevista por la ley. Diríjase a CEDRO (Centro Español de Derechos Repográficos, www.cedro.org) si necesita fotocopias o escanear algún fragmento de esta obra. 


\title{
HACIA LA NUEVA GOBERNANZA. RETOS PARA UNA BUENA GESTIÓN E IMPLEMENTACIÓN DE PLANES ESTRATÉGICOS EN TERRITORIOS RURALES Y CIUDADES PEQUEÑAS. EL CASO DE CATALUÑA
}

\author{
Marta Pallarès-Blanch \\ Centre de Desenvolupament Rural Integrat de Catalunya (CEDRICAT) \\ Marta.pallares@gmail.com \\ Marta Planas Vilafranca \\ Centre de Desenvolupament Rural Integrat de Catalunya (CEDRICAT) \\ marta.planas@cedricat.cat \\ Antoni F. Tulla i Pujol \\ Departamento Geografia. Universitat Autònoma de Barcelona \\ Antoni.tulla@uab.cat
}

RESUMEN

El contexto actual de recisión económica generalizada demanda una economía de recursos y comporta una revisión profunda del sistema de crecimiento económico dominante. La adopción de acciones dirigidas a promover la resiliencia, es decir, la capacidad de afrontar la adversidad para salir reforzado de ella es una primera medida. El nuevo escenario está desencadenando, en consecuencia, la renovación de los modos tradicionales de organizar las políticas públicas, entre las que se incluyen los planes estratégicos; planes que han evolucionado en respuesta a las nuevas necesidades de organización socioeconómica y política.

La crisis en las haciendas locales lleva a los gobiernos locales ante una disyuntiva: obviar la situación y seguir con la misma dinámica, que conduce a la devaluación de las instituciones y a la decadencia de la ciudad, o bien generar procesos de nueva gobernanza para reactivar la ciudad y salir del callejón sin salida. 
Los planes estratégicos de "segunda generación" son un buen instrumento de organización social para iniciar la transición desde la gobernación actual hacia la nueva gobernanza. Mantener el sistema de gobernación actual lleva a una secuencia decadente, ya que la provisión y gestión de los servicios públicos no puede satisfacer a la ciudadanía, tal y como estaba acostumbrada. Esta relación de la institución con el ciudadano como usuario-cliente ya no es posible. La relación de la institución con la ciudadanía se presenta como viable es la de la interdependencia, ser mutuamente responsables, compartiendo un conjunto de principios y objetivos comunes. En la gestión de las interdependencias, la confianza, a nivel individual y colectivo, es la principal aliada para desarrollar la capacidad de adaptación y para posibilitar la innovación. Para ello, se necesitan nuevos instrumentos de carácter social, que son los que van a permitir alcanzar la nueva gobernanza. Estos son los retos actuales para una óptima gestión e implementación de los planes estratégicos: ejercer un liderazgo representativo, procurar una administración relacional, fomentar la ciudadanía activa, conseguir el compromiso de la ciudadanía, impulsar la cooperación entre actores para lograr la cohesión social y desarrollar proyectos de futuro. Dichos retos a afrontar son mayores aun en territorios rurales y ciudades pequeñas.

La Fundación CEDRICAT ha venido realizando planificación estratégica desde el inicio de su actividad, en 2002, para el diseño, realización y seguimiento de actuaciones para el desarrollo rural. En base a la evolución constatada en la realización de planes estratégicos durante los últimos años, y coincidiendo con el veinticinco aniversario en Cataluña del primer plan estratégico, en esta comunicación se exponen cuáles son les retos para la gestión e implementación de dichos planes específicamente en territorios rurales y ciudades pequeñas. La comunicación incide específicamente en los factores que pueden facilitar y los que pueden obstaculizar el proceso de transición desde la gobernación actual a la nueva gobernanza y cuáles son las condiciones de base para qué la planificación tenga las repercusiones esperadas en el territorio.

Palabras clave: Nueva gobernanza, planes estratégicos de segunda generación, Solsona-co, áreas rurales, cooperación.

FROM CURRENT GOVERNMENT TO THE NEW GOVERNANCE. CHALLENGES FOR GOOD MANAGEMENT AND IMPLEMENTATION OF STRATEGIC PLANNING IN RURAL TERRITORIES AND SMALL CITIES

\section{AbSTRACT}

The current context of widespread economic rescission demands an economy of resources and involves a thorough review of the dominant economic growth system. The adoption of actions to promote resilience, ie the ability to face adversity to emerge stronger from it is a first step. The new scenario is triggering 
therefore the renewal of traditional ways of organizing public policies, among which include strategic plans, plans that have evolved in response to changing needs of socioeconomic and political organization.

The crisis in local finances leads to local governments face a choice: ignore the situation and continue with the same dynamic that leads to the devaluation of the institutions and the decline of the city, or build new governance processes to revive the city and out of the impasse.

The strategic plans of "second generation" are a good instrument of social organization to begin the transition from the current government to the new governance. Keep the current governance system leads to a decaying sequence, since the provision and management of public services cannot meet the public, as was customary. This relationship between the institution and the citizen as client-user is no longer possible. The relationship between the institution and the public is presented as feasible is the interdependence, be mutually accountable, sharing a set of common principles and objectives. In interdependencies management, trust, individual and collective level, is the main ally to develop resilience and to enable innovation. To do this, we need new social tools, which are the ones to help achieve the new governance. These are the current challenges for optimal management and implementation of strategic plans: exercise leadership representative, seek a relational management, encourage active citizenship, get the commitment of citizens, promote cooperation between actors and achieve social cohesion and develop future projects. These are challenges to be faced, even if possible, higher in rural areas and small towns.

CEDRICAT Foundation has been conducting strategic planning from the beginning of its activity, in 2002, for the design, implementation and monitoring of rural development interventions. Based on the changes observed in the implementation of strategic plans in recent years, coinciding with the twentyfifth anniversary in Catalonia's first strategic plan, in this submission sets out what challenges them to the management and implementation of these plans specifically in areas rural and small towns. Communication affects specifically the factors that can facilitate and those that can hinder the process of transition from the current government to the new governance and what are the basic conditions for which planning has the expected impact on the territory.

Key Words: New Governance, Second Generation of Strategic Planning, Solsona-co, rural areas, cooperation.

\section{INTRODUCCIÓN}

El actual contexto de recesión económica generalizada está forzando la renovación de las formas tradicionales de organizar las políticas públicas entre las que se incluyen los planes estratégicos. Los mismos planes estratégicos han evolu- 
cionado en respuesta a las nuevas necesidades de organización socioeconómica y política. El principal valor añadido en los planes estratégicos de "segunda generación" es la inclusión de la ciudadanía en procesos participativos.

En los nuevos planes estratégicos, el proceso que se inicia para la realización, coordinación, consulta y futura implementación del proyecto es la herramienta fundamental. Como se ha dicho recientemente (Ganau, 2013) los de "primera generación" eran más "planes" que "estratégicos". A menudo se reproducía la misma fórmula en territorios con necesidades diferentes o bien sobresalían casos de ciudades, que disponiendo de una fuerte proyección, planteaban un conjunto de medidas para reforzar una acción emblemática, generalmente de tipo arquitectónico. En sustitución de esa manera de idear el futuro de una ciudad o de un territorio, el escenario actual exige acciones dirigidas a reforzar la resiliencia, es decir, la capacidad de afrontar la adversidad saliendo reforzados a partir de las propias herramientas. No es casualidad que el término resiliencia provenga del campo de la psicología, ya que cada vez más los aspectos relacionales y de la inteligencia emocional son considerados cruciales en el desarrollo de procesos de todo tipo.

El escenario actual demanda una economía de recursos y comporta una revisión profunda del sistema de crecimiento económico dominante. Ante el aumento de las desigualdades en todas las escalas, el desarrollo que satisface las necesidades del presente, sin comprometer las de las generaciones futuras, se presenta como la manera más sensata de reconducir el modelo de organización socioeconómica. Por ello, los aspectos económicos forman, junto con los aspectos ambientales y los sociales, los tres pilares del desarrollo sostenible. En relativamente poco tiempo, hemos presenciado el acercamiento hacia el realismo de estos términos que nos parecían teóricos unos años atrás. La globalización ha acelerado los acontecimientos y afrontarlos requiere todo un cambio de planteamiento y de transformación de las viejas estructuras, tanto a nivel individual como colectivo. En ambos niveles, la confianza es el principal aliado para desarrollar los instrumentos que pueden llevar a la innovación.

\section{Metodología y fuentes}

La metodología de trabajo aplicada para la realización de la presente comunicación es de tipo cualitativo. Los resultados de campo corresponden al trabajo de diagnosis sobre "El potencial de Desenvolupament Económic Local de l'Alt Pirineu i Aran" que se realizó en 2008 y que consistió en 32 entrevistas semiestructuradas a agentes del desarrollo local de este territorio (AODL, técnicosas de promoción económica, gerentes de Grupos de Acción Local, director del Ecomuseu de les Valls d'Àneu, profesores de las Escuelas de Capacitación Agraria, técnicos-as o directores-as de las Oficinas Comarcales de Agricultura, gerentes de Consells Comarcals, directores de Parques Naturales y Parque 
Nacional, entre otros). Tomamos el caso de la región de montaña, Alt Pirineu i Aran, para ejemplificar la evolución de la planificación estratégica en áreas rurales y remotas de Catalunya. Se interpretan los resultados en el marco de las aportaciones teóricas más recientes sobre la temática de la planificación estratégica y el desarrollo local y rural. Se ha procurado consultar publicaciones basadas en territorios cercanos al ámbito de estudio. La revisión bibliográfica incluye también los estudios e informes de planificación local realizados en el ámbito de estudio, en los que se ha participado en algunos casos de forma directa.

\section{GOBIERNO Y PLANIFICACIÓN ESTRATÉGICA}

Desde ya hace un tiempo sabemos que el desarrollo local depende de la capacidad de organización y acción de una sociedad, esto es, de la capacidad de articular los potenciales capitales humanos y capitales físicos hacia finalidades y objetivos de progreso ampliamente compartidos (Yori, 2000; Elizalde, 2003; Rodríguez, 2009). Al mismo tiempo, estos son los factores estructurantes para la construcción colectiva del desarrollo humano (Pascual, 2009)

Se ha constatado además que las ciudades que adoptan una política de colaboración abierta y relacional experimentan mayores avances (Pascual, 2009). Con frecuencia estas condiciones de apertura coinciden también con el hecho de ser ciudades que han iniciado procesos de decisión y visualización conjunta de una ciudad mejor para todos. La planificación estratégica es la herramienta que permite recoger estos procesos, vehicularlos y llevarlos al terreno de la implementación (Elizalde, 2003). Durante los años ochenta y noventa del siglo XX se generaliza la aplicación de planes estratégicos por parte de los gobiernos locales, en aquellos territorios más decididos a incidir en su propio futuro (Precedo et al., 2010). En Cataluña, la propagación de la planificación estratégica ha sido pionera por la iniciativa de Barcelona que ha influenciado decisivamente a los municipios de su área metropolitana y también de ciudades medias (Ganau et. al., 2003). La realidad rural es otra cosa, sobre todo cuando se trata de las áreas más alejadas de la influencia de la ciudad de Barcelona.

\subsection{Planes Estratégicos de Primera Generación}

La finalidad de lo que se ha venido a llamar Planes Estratégicos de Primera Generación se centró en el desarrollo económico, generalmente a escala municipal. Se planteaban proyectos específicos y tangibles partiendo de un liderazgo corporativo que propiciaba más que una cooperación una competencia entre ciudades.

Con demasiada frecuencia, una vez conseguidos, más o menos, los objetivos se abandonaba el seguimiento del proceso llevando a la desimplicación y posteriormente decepción o desgaste de la participación ciudadana (Ganau et. 
al., 2003). A pesar de ello, dos estudios evaluativos de la Diputación de Barcelona, en 1998 y en 2006 elaborados por consultoras diferentes llegan a las mismas conclusiones: los planes estratégicos han servido poco para impulsar directamente los proyectos clave, en cambio, han mejorado la confianza y el compromiso entre actores, dando lugar a una mejora de los procesos de organización social. (Pascual, 2009).

\subsection{Planificación estratégica en las áreas rurales}

En el ámbito rural, la adopción de la planificación estratégica como una práctica generalizada ha llevado algo más de tiempo y empezó de forma bastante relacionada con aspectos sectoriales como la planificación a través de los aspectos ambientales (Agendas 21) y a través de la prospección del sector turístico (Planes Directores) que, con toda lógica, son las principales potencialidades de los territorios rurales. Los programas europeos LEADER han sido quizás la herramienta de planificación más consolidada entre las instituciones, con impactos diferentes según el dinamismo de los territorios (Esparcia et. al., 2000, Pallarès-Blanch et. al. 2011).

En los últimos diez años la planificación estratégica se ha ido implantando en el hacer cotidiano de muchos municipios o comarcas rurales. Sus especificidades se centran de una parte, en la escasa masa crítica que caracteriza a los municipios rurales, presentando claros límites para la participación e implicación de la ciudadanía. Esto da lugar a que sean pocos y siempre los mismos los ciudadanos los que se ofrecen para formar parte de los procesos de debate, conduciendo en bastantes casos a situaciones de saturación. Otra limitación, ésta compartida con las zonas urbanas aunque se da de forma más acusada en las áreas rurales, es la desatención en el seguimiento del proceso, expresándose en una pobre asignación en recursos técnicos y económicos (Tolón y Lastra, 2009). De otra parte, las poblaciones rurales tienen su lado ventajoso para la planificación estratégica, especialmente en aquellas áreas en las que el capital social es activo y muestra altos grados de consenso, ya que las pequeñas dimensiones de la población, que no del territorio, facilitan el acceso a amplios sectores de la ciudadanía (Pallarès-Blanch, 2012). Si los municipios son las unidades básicas del territorio y sus ayuntamientos las instituciones político administrativas más cercanas al pueblo, esto es aún más cierto en los pequeños municipios (Aldomà, 1999)

\subsection{Tendencias y características del desarrollo local en el Alt Pirineu i Aran}

Durante los años ochenta y noventa del s. XX la planificación territorial en Catalunya avanza, aunque el Plan Territorial General no se aprueba hasta 1995. Aparte de la planificación sectorial (infraestructuras, educación, sanidad etc.), crece la importancia del aspecto ambiental fuera del territorio metropolitano 
con los planes especiales de interés natural (PEIN). En las zonas de montaña se iniciaron los Plans Comarcals de Muntanya (PCM) (1990), impulsados por la Llei de Muntanya (1983). Los PCM son la primera experiencia de planificación y gestión territorial a nivel de comarca, unidad territorial que en Catalunya tiene una fuerte componente identitaria. Los puntos fuertes de los PCM son la exhaustividad de las diagnosis, la incorporación de la consulta a los agentes del territorio y la transversalidad de las actuaciones presupuestadas (generalmente en infraestructuras y equipamientos) por los diferentes organismos competentes (Departamento de Agricultura, Medio Ambiente, Política Territorial, Turismo, Cultura, Trabajo etc.). Gracias a estas actuaciones en los años noventa se realizan las principales inversiones en infraestructuras de la historia de los municipios rurales, especialmente en las áreas de montaña (carreteras locales, red de aguas residuales, potabilización, nuevas captaciones y depósitos de agua, equipamientos sanitarios etc.). Los puntos débiles de los PCM son la escasa incorporación del enfoque del desarrollo endógeno y su marcado carácter descendente, siendo decisiones tomadas des del gobierno autonómico, es decir, desde la ciudad.

En los mismos años noventas surgen algunos planes estratégicos de abasto supracomarcal, como fue el caso de la región o ámbito territorial del Alt Pirineu i Aran (IEMB, 1998; Generalitat de Catalunya, 1998). Sus características corresponden a las de los llamados Planes Estratégicos de Primera Generación (Pascual, 2009 y 2013): las diagnosis son ya más concisas -el análisis socio-económico estaba ya disponible en diversas publicaciones previas-, se presenta una jerarquización de las propuestas articulada a través de medidas generales y actuaciones concretas y las consultas populares no son solo consultas sino que también contemplan el debate. Coincide en esta fase el inicio de la implantación del correo electrónico y de las páginas web, que se incorporan como medios de difusión aumentando el impacto mediático y con ello la sensibilización social. A pesar de que los objetivos de ambos planes incluyen líneas de actuación para la protección y gestión del patrimonio cultural y natural, las acciones que han tenido mayor consecución fueron las del ámbito socioeconómico, profesionalizando la oferta turística y diversificando la economía agraria. A pesar del énfasis en las propuestas respecto a los documentos de planificación anteriores, la falta de compromiso de las instituciones para la ejecución de las propuestas, muy generalistas, conduce al escepticismo de la población hacia este tipo de dinámicas, que por aquel entonces eran lideradas por agentes externos al territorio (Ganau et. al. 2003). Dado el escaso alcance de actuaciones concretas, la principal utilidad después de la realización de estos planes estratégicos fue la contribución en desarrollar un discurso, en buena parte basado en las consultas a la población, que fue penetrando en el imaginario colectivo hasta verse reflejado una década más tarde en la ejecución de algunos proyectos. 
Los Planes Territoriales parciales -por ámbitos territoriales- (2006-2010) recogen estas diagnosis y líneas estratégicas aun sin embargo, con una fuerte orientación urbanística-económica. En paralelo, en esta última década es cuando han surgido o se han consolidado instituciones y entidades de actuación transversal tanto de carácter público como privado, que reflejan el impulso del enfoque del desarrollo local en estas áreas. Se trata tanto de entidades gubernamentales, como de centros transferencia tecnológica, asociaciones, fundaciones o consultoras dedicadas a la dinamización y asistencia técnica de procesos estratégicos. En la demarcación de Lleida, la parte más rural de Catalunya, podemos citar la creación del Ecomuseu de les Valls d'Àneu (1994), el Centre Tecnològic Forestal de Catalunya (CTFC) (1996), la Fundació Centre de Desenvolupament Rural Integrat de Catalunya (CEDRICAT) (2001), l'Institut de Promoció i Desenvolupament de l'Alt Pirineu i Aran (IDAPA) (2003), Socarrel SL (2004), Rurbans (2004), Montanyanes SL (2006), Arada (2007), Red de Museus del Pirineu (2008), entre otros. Así mismo los Parques Naturales toman una orientación mucho más integrada entre conservación y desarrollo local, constituyendo uno de los agentes más activos en el impulso de iniciativas de promoción local (Pallarès-Blanch, 2012).

\subsection{Balance de diez años de planificación estratégica en el Alt Pirineu i Aran}

Diez años después de la presentación de los planes estratégicos de ámbito regional (IEMB, 1998; Generalitat de Catalunya, 1998) la Fundación CEDRICAT realiza por encargo de IDAPA una diagnosis sobre el potencial de desarrollo económico local del ámbito territorial del Alt Pirineu i Aran (IDAPACEDRICAT, 2008) con los siguientes resultados:

\subsubsection{Los puntos fuertes}

En síntesis son: (1) Las potencialidades de un territorio con elevado patrimonio natural y cultural bien conservado y poco explotado, (2) La existencia de iniciativas de producción y transformación agroalimentaria con productos con valor añadido y ecológicos, (3) La consciencia de la necesidad de vincular turismo, naturaleza y cultura en una estrategia de futuro, (4) El avance en la implantación de internet de banda ancha, (5) La cooperación entre las Oficinas Comarcales del Departamento de Agricultura y las Escuelas de Capacitación Agraria, especialmente en relación con las necesidades formativas, (6) La voluntad de los técnicos/cas de establecer redes de transmisión de información e intercambio de experiencias, por ejemplo impulsando la Xarxa Museus de l'Alt Pirineu, (7) La mayor voluntad de colaborar por parte de las entidades de ámbito supracomarcal, en las que hay más enfoque técnico que político, (8) La labor de los AODL jugando un papel clave en la concreción de las ideas y la creación, puesta en funcionamiento y mantenimiento de las actuaciones de desarrollo económico local. 


\subsubsection{Los puntos débiles}

Las respuestas acusan, en primer lugar, la escasa cooperación entre territorios $\mathrm{y}$ entre instituciones, tanto a nivel horizontal como vertical. En segundo lugar, reflejan la limitada implantación y avance de las estrategias de desarrollo local. En tercer lugar, incluyen las características del medio (clima, relieve, distancias, núcleos pequeños y dispersos, envejecimiento de la población etc.) como elementos que frenan el desarrollo económico (escasas inversiones en infraestructuras, menores servicios para la población, distancias hacia los centros de formación y educación etc.).

La escasa cooperación territorial, se menciona como una forma de "trabajar fuera de red" o teniendo un "carácter puntual". Este trabajo individualizado, explican las respuestas, da lugar a solapamientos, por ejemplo, entre marcas de productos artesanos y marcas turísticas. La atomización es una tendencia que se reconoce tanto entre el sector público como el empresarial, entre los técnicos y entre los políticos y también entre diferentes administraciones por niveles competenciales y por sectores temáticos (principalmente agricultura, medio ambiente, cultura, trabajo y política territorial).

El desconocimiento de la metodología de desarrollo local, en concepto, método y práctica, conduce a omitir sus potencialidades en aspectos como la generación y conservación de puestos de trabajo, la fijación de la población en el territorio, la mejora de la calidad de vida y una mayor igualdad de oportunidades.

El débil desarrollo de las redes de cooperación en materia de desarrollo local en esta área occidental del Pirineo catalán, se muestra como causa y consecuencia a la vez de las características de su medio (poca oferta formativa local para profesionales y largas distancias con los centros urbanos para acceder a ella, carencia de instituciones que ofrezcan espacios de diálogo y contraste de ideas, largas distancias también con los territorios con iniciativas interesantes). El vacío en materia de desarrollo local se percibe y expresa como "la necesidad de otras actuaciones de carácter más social a parte de los PCM".

Los puntos débiles detectados a partir de las entrevistas se centran en, por un lado, la insuficiente disponibilidad de recursos económicos por parte de las administraciones locales para dar apoyo a iniciativas con potencial y para consolidar a los Agentes de Ocupación y Desarrollo Local (AODL) y por otro lado, en la falta de prioridades y estrategias a nivel político.

\subsubsection{Conclusiones}

En suma, se identifica en esta diagnosis una clara necesidad de actuaciones para fortalecer el capital social por un lado, y por otro lado la necesidad de construir una gobernanza que engrase la coordinación vertical y horizontal.

A pesar de identificarse el siguiente circulo vicioso: $\rightarrow$ Pérdida de capital humano y capital intelectual $\rightarrow$ Mínimo despliegue de políticas socio-económi- 
cas $\rightarrow$ Falta de seguimiento político y continuidad de las acciones $\rightarrow$ Crisis del sistema de organización $\rightarrow$ Falta de puestos de trabajo cualificados $\rightarrow$ (se reinicia la secuencia), estos resultados indican un gran cambio comparados con los que se obtuvieron en las diagnosis de 1998 o anteriores, donde el énfasis en los discursos se focalizaba en las limitaciones del medio y en la falta de inversiones por parte de las administraciones superiores.

Se desprendió del análisis de los resultados de las entrevistas realizado en el año 2008 que las acciones prioritarias y estratégicas en el Alt Pirineu i Aran tenían que centrarse en el fomento a la creación de puestos de trabajo calificados y en retener y atraer a la población joven, en ofrecer formación especializada a nivel sectorial y también a nivel de implementación de políticas de desarrollo local, en establecer protocolos de relación interinstitucional y sistemas de cooperación entre instituciones, estimulando la capacidad de negociación. Estas acciones tenían que formar parte de acciones transversales encaminadas al fortalecimiento del capital social y al desarrollo de una nueva gobernanza (Pallarès-Blanch, 2009; Pallarès-Blanch, 2012).

En perspectiva, valoramos que en los años noventa además de las inversiones en infraestructuras, servicios y equipamientos, eran necesarias políticas que incidieran en los puntos críticos de este territorio: la debilidad de su tejido social y la pobreza de su administración relacional.

\section{EMERGENCIA DE LA NUEVA GOBERNANZA}

El concepto de Gobernanza se extiende en Europa a raíz de la publicación en 2001 del Libro Blanco para la Gobernanza Europea (CCE, 2001). El documento es fruto de la constatación de que la eficacia y la legitimidad de los gobiernos democráticos se fundamentan, cada vez más, en la calidad de la interacción entre éstos y las organizaciones empresariales y sociales, así como en la buena gestión de las relaciones entre los distintos niveles de gobierno. El concepto de gobernanza o nueva gobernanza trata de un modo de gobernar basado en la horizontalidad y el acuerdo relacionado con lo supranacional. Es muy significativo comprobar como las puntos críticos que se identificaron en la diagnosis sobre el potencial del desarrollo económico local en el Alto Pirineu i Aran, un territorio rural y de montaña concreto de la UE, correspondan a la justificación de los postulados del Libro Blanco para la Gobernanza Europea.

\subsection{Los principios de la Nueva Gobernanza}

Los instrumentos clave per a la nova governança son: 1) Ejercer un liderazgo representativo, 2) Procurar una administración relacional, 3) Fomentar la ciudadanía activa, participación e implicación, 4) Conseguir el compromiso de la ciudadanía, 5) Impulsar la cooperación entre actores, 6) Lograr cohesión social y desarrollar proyectos de futuro (Pascual, 2009). 


\subsection{Planes Estratégicos de Segunda Generación}

Una de las principales herramientas para incidir en un proceso social organizado y planificado son los planes estratégicos (Yori, 2000, Gutiérrez-Rubí, 2012). Las características específicas de los Planes Estratégicos de Segunda Generación (PESG) son, en primer lugar, la mayor intangibilidad de los proyectos, incidiendo en temas como educación, cultura, innovación, inclusión etc. En segundo lugar, la capacidad de organización y acción del territorio no es solo un apoyo al proceso estratégico sino una finalidad de actuación. En tercer lugar, se parte de un liderazgo relacional en lugar de un liderazgo corporativo (Pascual, 2009).

La mayor intangibilidad viene dada por el remplazo del paradigma de desarrollo económico por el del desarrollo humano que incluye el capital ético (léase los valores de la ciudadanía) y el capital social, entendido como la capacidad de generar tejido organizativo, empresarial y social para fines relacionados con el bien común. En este sentido, el capital social y el capital ético, junto con las redes de los actores son factores clave para la creación de desarrollo endógeno en los territorios, dado que las relaciones se establecen en base a intereses y se sustentan en valores y actitudes (Matheu, 2013). Dentro del capital social, el liderazgo relacional de uno o más actores, entendido como la "capacidad de implicar" los actores institucionales clave (Subirats, 2012), juega un papel decisivo porque de él depende la capacidad de movilización del territorio (Brugaroles, 2012).

Los PESG incluyen además los objetivos de profundizar en la democracia, la participación y deliberación con la ciudadanía, un mayor énfasis en calidad frente a la cantidad, la reforma de la administración pública y la colaboración intermunicipal y regional. En este sentido, el contexto de la sociedad red o sociedad del conocimiento supone un gran cambio porque propicia que el municipio, como unidad relacional, forme redes con ámbitos superiores para reforzar la autonomía municipal (Gutiérrez-Rubí, 2012). Estas redes de actores han de ser capaces de articular diferentes esfuerzos encaminados a incrementar capacidades sociales que den respuesta a los retos urbanos y territoriales (Yori, 2000).

Otro factor importante en la estrategia territorial de segunda generación es el compromiso de acción, partiendo del reconocimiento de que los intereses generales se componen de procesos de conflicto, negociación y consenso entre los diversos actores involucrados. La acción tiene que venir impulsada por una actitud abierta a la innovación, a la integración social y cultural y a la inserción en estrategias territoriales más amplias. Es más, la habilidad de una ciudad o un territorio para posicionarse en el futuro estriba en gran medida en la capacidad de articulación de las políticas regionales con las locales. Por esta razón es clave en el proceso saber organizar un espacio público deliberativo. 
Otro elemento esencial en los PESG es el reconocimiento del papel de la identidad y del sentimiento de pertenencia con la ciudad y la región en el desarrollo local (Pallarès-Barberà et. al., 2004).

A grandes rasgos, estos son los principios de la estrategia territorial en el marco del gobierno relacional. En este marco, la planificación estratégica emerge como un instrumento de gobernanza territorial que articula una serie de elementos intangibles y de relaciones en un proceso de organización de la ciudad o de un territorio para responder colectivamente (Yori, 2000). Al igual que el concepto de desarrollo local, no hay un modelo único de gobernanza sino que ésta es multifacética y plural. La nueva gobernanza persigue la eficiencia adaptativa y exige flexibilidad, experimentación y aprendizaje por prueba error. Por este motivo, parte del éxito del discurso de la nueva gobernanza es porque no bebe de una fuente única. Al mismo tiempo, al ser un concepto emergente y abierto adolece de imprecisión semántica, hecho que propicia su idealización (Closa, 2003).

\subsection{Los Planes Estratégicos de Segunda Generación en las áreas rurales y ciudades pequeñas. Ejemplo del Plan Director de Promoción Económica de Solsona (2012-2020)}

Al margen de las limitaciones del concepto de gobernanza, la filosofía de los PESG contiene elementos adecuados para iniciar procesos de decisión colectiva en áreas rurales por responder a un enfoque más amplio que el del desarrollo económico con la adopción del enfoque del desarrollo humano. El carácter adaptativo y relacional de este enfoque incluye el planteamiento holístico del concepto de desarrollo rural neo-endógeno, hasta ahora tratado de una forma bastante localizada. En este sentido, la evolución de la planificación estratégica favorecerá las áreas rurales por la intangibilidad de sus objetivos, que pueden adaptarse mejor a la diversidad que caracteriza las áreas rurales.

El proceso iniciado con la realización del Pla Director de Promoció Econòmica de Solsona 2012-2020 (Solsona-co) expresa esta transformación mostrando una elevada capacidad de reacción por parte de las instituciones locales de Solsona y comarca, ofreciendo el marco en el que desarrollar una visión conjunta basada en el conocimiento de los hechos, fomentando el ejercicio de la responsabilidad colectiva y creando un espacio de diálogo entre los agentes públicos y privados. Hasta el momento del proceso Solsona-co ha mostrado ser una óptima herramienta de cooperación y participación de la ciudadanía con un manifiesto compromiso de todos; agentes, instituciones y asociaciones para construir un futuro mejor para la ciudad de Solsona, capital del Solsonès. Los fundamentos sobre los que se ha generado el proceso son los de compartir un conjunto común de principios y objetivos con la premisa de que "gracias al éxito de los demás yo obtengo éxito". 


\subsubsection{Los puntos fuertes}

La ciudadanía de Solsona, como ciudad y como capital comarcal, revela un alto nivel de implicación. Se ha contado con una participación activa por parte de los actores y agentes (públicos y privados) más relevantes del territorio durante todo el proceso (diagnóstico, establecimiento de los objetivos, definición y priorización de las acciones, seguimiento y aplicación de algunas acciones). La ciudadanía activa, el compromiso de los ciudadanos y la cooperación entre los actores son la mitad de los requisitos indispensables para afrontar el cambio en la dirección recomendable.

\subsubsection{Los puntos débiles}

Los elementos críticos del proceso a reconducir radican en la necesidad de una mejor gestión de los imprevistos y en evitar situaciones de duplicidad representativa. Lamentablemente no es inusual en el actual contexto de crisi, que el escenario socioeconómico de partida, en este caso abril de 2012, se agrave por el cierre de empresas. El cierre de la fábrica TRADEMA, de tablones de conglomerado, en diciembre de 2012 supuso la pérdida de 126 puestos de trabajo directos y 160 indirectos. Ante esta nueva situación se plantearon acciones específicas en el marco de Solsona-co. Al mismo tiempo, desde el Ayuntamiento de Solsona y con la colaboración de agentes públicos y privados, se elaboró un Plan de choque específico. En este sentido, hubiera sido deseable una mayor integración entre ambas acciones.

Estas situaciones de duplicidad no corresponden con dos aspectos fundamentales de la nueva gobernanza, la del ejercicio de un liderazgo representativo bien visible y la de procurar una administración relacional, es decir transparente en las acciones y los comunicados.

\section{Retos PARA LA NUEVA GOBERNANZA EN ÁREAS RURALES Y CIUDADES PEQUEÑAS}

Los principales retos para la implantación de procesos estratégicos en el marco de una nueva gobernanza en áreas rurales y ciudades pequeñas residen, en primer lugar, en la necesidad de una apuesta clara a nivel institucional en destinar recursos técnicos y económicos y una colaboración puntualizada entre técnicos y políticos. Es importante recalcar este aspecto porque los procesos de planificación con una elevada participación de los agentes resultan en general más lentos y exigen mayores esfuerzos de coordinación. Un segundo elemento a asegurar es la existencia de un liderazgo político claro a la hora de sumar esfuerzos e implicar otros agentes y actores, entendiendo la acción de liderazgo como la capacidad de identificar e integrar los diferentes intereses en un proyecto común. Las áreas rurales en este sentido tienen un aprendizaje a desarrollar porque en ellas los liderazgos tienden a ser corporativos o bien cuestionados. En este sentido, las políticas de inclusión tendrán que ejercer un 
papel importante y más integrador (valga la redundancia), introduciendo otros elementos identitarios más allá del aspecto étnico. Por otro lado, la recomendación de integrar todas las entidades del territorio que desarrollan actividades de desarrollo local y de promoción económica en un único órgano de seguimiento y evaluación, a fin de aprovechar los conocimientos y las experiencias, puede presentar ciertas facilidades en las áreas rurales por su menor número y complejidad.

Resaltamos dos elementos específicos a tener en cuenta como aspectos frágiles o debilidades las áreas rurales. Por un lado, la debilidad del tejido social que caracteriza a muchas de estas áreas puede dificultar en gran medida la consecución de un proceso ampliamente representativo. En este sentido, creemos que desarrollar acciones específicas para la restitución y el fortalecimiento del tejido social es aun una asignatura pendiente que habrá que superar. En algunas comunidades rurales, especialmente en el caso de los territorios más remotos, a pesar de producirse una cierta recuperación poblacional por la existencia de una movilidad de retorno, los efectos del despoblamiento han mermado la capacidad de movilización. En consecuencia es oportuno mencionar el importante avance que se ha realizado en los últimos años en el desarrollo de políticas de juventud y medio rural. Otra particularidad de las comunidades rurales y ciudades pequeñas son los elementos patrimoniales que ejercen un gran poder identitario. En la misma dirección anterior, las acciones de dinamización que se apoyan en los elementos identitarios ejercen un gran poder de vinculación de la población y de un modo muy transversal, ofreciendo valiosos factores de motivación y de cohesión social.

Finalmente, la cooperación entre territorios vecinos (en ocasiones en otros estados políticos) y la búsqueda de alianzas rural-urbanas se presenta como una de las líneas a impulsar en el marco de los PESG para lograr un mejor posicionamiento de las áreas rurales o ciudades pequeñas en este contexto de renovación.

BIBLIOGRAFÍA

ALDOMÀ, I. (1999): La crisi de la Catalunya rural. Una geografia dels desequilibri. Lleida, Pagès Editors

COMISIÓN DE LAS COMUNIDADES EUROPEAS (CCE) (2001): La gobernanza europea. Un libro blanco. COM (2001) 428 final, Bruselas, 25.7.2001.

CLOSA MONTERO, C. (2003): "El libro blanco sobre la gobernanza", Revista de Estudios Políticos (Nueva Época), Núm. 119.

ELIZALDE HEVIA., A. (2003): "Planificación estratégica territorial y políticas públicas para el desarrollo local", CEPAL Serie Gestión Pública, 29. Instituto Latinoamericano y del Caribe de Planificación Económica y Social (ILPES), Santiago de Chile, febrero de 2003. 
ESPARCIA, J.; NOGUERA, J. i PITARCH, M.D. (2000): "LEADER en España: desarrollo rural, poder, legitimación, aprendizaje y nuevas estructuras". Documents d'Anàlisi Geogràfica, $n^{o}$ 37: p. 95-113.

BRUGAROLES, C. (2012): "L'adaptació de les polítiques públiques en pro del territori" P3T, Journal Of Public Policies And Territories. Crisis and Local Policies, $\mathrm{n}^{\circ}$ 1, p. 43-61. http://www.politicsandterritories.com [Consultado: $16 / 05 / 13]$

Generalitat de Catalunya (1998): Pla Estratègic de les Comarques de Muntanya. Departament de Política Territorial i Obres Públiques. Subdirecció Acció Comarcal. Barcelona.

Institut d'Estudis Metropolitans de Barcelona (1998): Bases per al Pla Estratègic de l'Alt Pirineu i Aran. Barcelona.

IDAPA-CEDRICAT (2008): El potencial de Desenvolupament Económic Local de l'Alt Pirineu i Aran. Tremp-Solsona.

JIMÉNEZ HERNÁNDEZ, E. (2012): "Cambio activo o derrumbe forzoso en el mandato local" P3T, Journal Of Public Policies And Territories Crisis and Local Policies. $N^{o} 1$, p. 1-9. http://www.politicsandterritories.com [Consultado: 16/05/13]

MATHEU, X. (2013): "Governança multinivell i partenariat a la política europea de cohesió territorial", P3T, Journal Of Public Policies And Territories The territorial dimension of EU policy guidelines (EU2014-2020) $N^{o}$ 4, Winter, p. 1-7. http://www.politicsandterritories.com [Consultado: $16 / 05 / 13]$

GANAU CASAS, J.; MALLARACH ISERN, J. (2003): La planificació estratègica territorial a Catalunya Quinze anys d'evolució. Programa 7 Territoris. Pla Governamental CAT 21, Generalitat de Catalunya, Departament de la Presidència

GUTIÉRREZ-RUBÍ, A. (2012): La inteligencia de las multitudes para una mejor gobernanza P3T, Journal of Public Policies and Territories, Participation, Citizen control, Governance, $N^{o}$ 2, p. 40-49. http://www.politicsandterritories.com [Consultado: 16/05/13]

PALLARES-BARBERA, M.; TULLA, A.F.; VERA, A. (2004): "Spatial loyalty and territorial embeddedness in the multi-sector clustering of the Berguedà region in Catalonia (Spain)" Geoforum. Vol.35, nº 5, p. 635-649

PALLARÈS-BLANCH, M. (2001): La planificació estratègica a l'Alt Pirineu Català, Ponencia en el marco del Máster en Participación Ciudadana del Departament de Sociologia de la Universitat Autònoma de Barcelona.

PALLARÈS-BLANCH, M. (2012): "Natural Protected Areas Benefits and Rural/Local Development: A Sustainable Strategy in Remote Areas", En: M. Pallares-Barbera; P. Suau-Sanchez; R. Le Heron; M. Fromhold-Eisibith (editors) Globalising Economic Spaces, Uneven Development and Regional Challenges http://urbani-izziv.uirs.si/en/Specialissues/2012.aspx 
ISSN Print edition: 0353-6483, Online edition: 1855-8399. [Consultado: 14/05/13].

PALLARÈS-BLANCH, M.; PLANAS VILAFRANCA, M.; TULLA PUJOL A.F. (2010): "La atención a los temas del desarrollo rural desde la geografía y los estudios rurales", XV Coloquio de Geografía Rural "Territorio, paisaje y patrimonio rural" Cáceres, Universidad de Cáceres, 2010.

http://age.ieg.csic.es/geografia rural/Actividades\%20de\%20grupo/Documentos/XVColoquio Caceres\%202010/Comunicaciones/ponencialb.html [Consultado: 16-05-13]

PALLARÈS-BLANCH, M. (2009): The benefits of Nature Reserve Areas in local development: An opportunity to develop a sustainable strategy in peripheral areas a Prados, Maria José (eds) Naturbanization: New identities and processes for rural-natural areas. Taylor \& Francis Group, London. ISBN: 978-0-415-49000-9 (Hbk).

PASCUAL, J. M. (2013): "Introducció a la planificació estratègica de $2^{\mathrm{a}}$ generació". En: Seminari "La governança en les estratègies locals". Oficina Tècnica d'Estratègies per al Desenvolupament Econòmic (OTEDE), Diputació de Barcelona.

PASCUAL, J. M. i Tarragona, M. (2009): "Los ayuntamientos, gobiernos protagonistas en la sociedad contemporánea o sociedad en red". En: Estrategia territorial y gobierno relacional. Manual para la planificación estratégica de $2^{a}$ generación. Ed: Junta de Andalucía. Consejería de Gobernación.

PRECEDO LEDO, A.; OROSA GONZÁLEZ, J.J.; MÍGUEZ IGLESIAS, A. (2010): "De la planificación estratégica al marketing urbano: hacia la ciudad inmaterial" EUR, Vol. 36, no 108, p. 5-27.

RODRÍGUEZ COHARD, J. C. (2009): "Los procesos de desarrollo local desde la perspectiva europea: Génesis y transformación", Semestre Económico. Vol.12, nº 24 (Edición especial), p. 37-55, Medellín, Colombia.

SUBIRATS, J. (2012): "Lideratge i canvi d'època" Ponencia en el marco de la $9^{a}$ edición de Ordit, Programa de Liderazgo y Transformación Social. Fundació Jaume Bofill y Institut de Govern i Polítiques Públiques. Barcelona, junio, 2012.

TOLÓN BECERRA, A. y LASTRA BRAVO, X. (2009): "Planificación en los espacios rurales españoles Aplicación del modelo neo-endógeno para un desarrollo sostenible en las comarcas de metodología LEADER", Observatorio Medioambiental, vol. 12, 49-75.

YORY, C. M. (2000): "La planificación estratégica y la participación de los actores sociales locales", INTERAÇÕES, Revista Internacional de Desenvolvimento Local. Vol. 1, no 1, p. 31-40. 